\title{
Impacto dos Stents de Nova Geração no Tratamento de Enxertos Venosos Aortocoronários - Comparação Angiográfica e Ultra-sonográfica de Duas Séries First-in-Human: MGuard ${ }^{\circledR}$ vs. SESAME ${ }^{\circledR}$
}

\author{
Alaor Mendes ${ }^{1}$, José Ribamar Costa Jr. ${ }^{1}$, André Lima Brito ${ }^{1}$, Ricardo A. Costa ${ }^{1}$, Felipe Maia ${ }^{1}$, \\ Andrea Sousa Abizaid ${ }^{1}$, Áurea Chaves ${ }^{1}$, Fausto Feres ${ }^{1}$, Luiz Alberto Mattos ${ }^{1}$, \\ Amanda G. M. R. Sousa' ${ }^{1}$, J. Eduardo Sousa', Alexandre Abizaid ${ }^{1}$
}

\section{RESUMO}

Fundamento: As intervenções percutâneas em pontes de safena (PS) apresentam menores taxas de sucesso angiográfico e maior risco de complicações, pelo risco aumentado de embolização dos fragmentos da placa aterosclerótica friável. Com o advento dos dispositivos de proteção distal, observou-se maior eficácia e segurança do procedimento. Nosso objetivo foi comparar os stents MGuard ${ }^{\circledR}$ e SESAME $^{\circledR}$, dedicados ao tratamento de PS, nos primeiros 30 dias de evolução. Método: Estudo retrospectivo, comparando as variáveis angiográficas e ultra-sonográficas e a evolução clínica aos 30 dias de pacientes portadores de lesões em PS tratados com stent balão-expansível MGuard $^{\circledR}(\mathrm{n}=11)$ vs. stent auto-expansível $\operatorname{SESAME}^{\circledR}(n=14)$. Resultados: Os pacientes tratados com stents MGuard $^{\circledR}$ e SESAME $^{\circledR}$ demonstraram perfis clínicos superponíveis (média de idade, 69 anos; sexo masculino, $72 \%$; diabéticos, 28\%; tratados na vigência de síndrome coronária aguda, 44\%), idade dos enxertos venosos aortocoronários equivalentes $(9,7 \pm 6,2$ anos vs. $10,1 \pm 3,2$ anos; $p=0,80$ ) e PS com o mesmo grau de acometimento aterosclerótico. O sucesso angiográfico pós-procedimento foi alcançado em todos os casos, porém com tendência a melhor expansão do stent no grupo MGuard $^{\circledast}(87 \%$ vs. $68 \%$; p $=0,08)$. No seguimento clínico de 30 dias, os pacientes tratados com stent SESAME ${ }^{\circledR}$ apresentaram maior freqüência de eventos cardíacos maiores (dois casos de infarto do miocárdio não-Q e um caso de revascularização do vaso-alvo), comparativamente a nenhum evento no grupo MGuard ${ }^{\circledR}$. Conclusões: Nessa análise retrospectiva de pequena série de pacientes pré-selecionados de duas séries first-in-human, o stent balão-expansível

\section{SUMMARY}

Impact of New Generation Stents in the Treatment of Aortocoronary Venous Grafts - Angiographic and Ultra-sonographic Comparison of Two First-in-Human Series: MGuard ${ }^{\mathrm{TM}}$ vs. SESAME ${ }^{\mathrm{TM}}$

Background: Percutaneous interventions in saphenous vein grafts (SVG) result in lower rates of angiographic success and higher risk of complications, due to the increased risk of embolization of debris from the friable atherosclerotic plaque. With the advent of distal protection devices, improvements in efficacy and safety were achieved in the procedure. Our objective was to compare the MGuard ${ }^{\mathrm{TM}}$ and SESAME ${ }^{T M}$ stents, dedicated for SVG treatment, during the first 30 days of follow up. Methods: This is a retrospective study comparing angiographic and ultra-sonographic variables and clinical events within 30 days in patients with SVG lesions treated with MGuard ${ }^{\mathrm{TM}}$ balloon-expandable stents $(n=11)$ vs. SESAME ${ }^{T M}$ self-expandable stents $(n=14)$. Results: Patients treated with MGuard ${ }^{\mathrm{TM}}$ and SESAME $^{\mathrm{TM}}$ stents had similar clinical profiles (mean age 69 years; male gender, $72 \%$; diabetic, $28 \%$; treated for acute coronary syndrome, $44 \%$ ), equivalent age of aortocoronary venous grafts $(9.7 \pm 6.2$ years vs. $10.1 \pm 3.2$ years; $p=0.80)$ and SGV with the same degree of atherosclerotic impairment. Post-procedure angiographic success was reached in all cases, however with a tendency towards better stent expansion in the MGuard ${ }^{\mathrm{TM}}$ group $(87 \%$ vs. $68 \%$; p = 0.08). Over the 30-day clinical follow-up, patients treated with the SESAME ${ }^{\mathrm{TM}}$ stent had higher frequency of major cardiac events (two cases of non-Q myocardial infarction 
MGuard ${ }^{\circledR}$ mostrou-se superior ao stent auto-expansível SESAME $^{\circledR}$ nos primeiros 30 dias de acompanhamento clínico.

DESCRITORES: Contenedores. Angioplastia translumial percutânea coronária. Ponte de artéria coronária. Veia safena.

$\mathrm{H}$ istoricamente, as intervenções coronárias percutâneas (ICPs) em pontes de safena (PS) têm apresentado menores taxas de sucesso angiográfico e maior risco de complicações intraprocedimento (incluindo os fenômenos de slow flow e no-reflow), em decorrência de embolização de material aterosclerótico para a microvasculatura distal, resultando no incremento dos índices de infarto do miocárdio (IM) durante a fase intra-hospitalar e pior prognóstico imediato e tardio ${ }^{1,2}$.

Estudos recentes com o uso de dispositivos de proteção da microcirculação distal (filtros e sistemas de aspiração) demonstraram redução da taxa de eventos agudos, com benefícios inclusive a longo prazo $^{3}$. Entretanto, o uso de tais dispositivos adiciona tempo e custo ao procedimento, e, em alguns casos, requer a utilização de cateteres de maior calibre (> $6 \mathrm{Fr}$ ).

Diante das limitações dos sistemas atuais, pesquisas recentes têm focado no desenvolvimento de stents capazes de prevenir a microembolização distal durante o tratamento de PS, com o propósito de melhorar os resultados da ICP nesse complexo cenário.

No presente estudo, reportamos a comparação entre dois stents de nova geração $\left(M^{\prime}\right.$ uuard $^{\circledR}$ e SESAME $\left.{ }^{\circledR}\right)$ dedicados ao tratamento de PS.

\section{MÉTODO}

\section{Protocolo do estudo}

A presente análise foi realizada de maneira retrospectiva, comparando de forma não-randomizada a evolução intra-hospitalar e aos trinta dias de pacientes portadores de lesões de novo (estenose $\geq 50 \%$ ) em PS, tratados de maneira consecutiva com stents SESAME ${ }^{\circledR}$ vs. MGuard ${ }^{\circledR}$ em nossa instituição (Instituto Dante Pazzanese de Cardiologia - São Paulo, SP) durante a realização de duas séries first-in-human de 2005 a 2008.

Descrição do stent MGuard ${ }^{\circledR}$ (InspireMD Ltd.,Tel Aviv, Israel)

Plataforma e polímero: Stent não-farmacológico, balão-expansível, de aço inoxidável, com hastes de baixo perfil $(150 \mu \mathrm{m})$, montado sobre um cateter-balão semicomplacente compatível com o cateter 6 Fr. O polímero durável é composto por fina malha de fibras and one case of revascularization of the target vessel), compared with no events in the MGuard ${ }^{T M}$ group. Conclusions: In this retrospective analysis of a small series of pre-selected patients from two first-in-human series, the MGuard ${ }^{\mathrm{TM}}$ balloon-expandable stent showed to be superior to the SESAMETM self-expandable stent over the first 30 days of clinical follow-up.

DESCRIPTORS: Stents. Angioplasty, transluminal, percutaneous coronary. Coronary artery bypass. Saphenous vein.

de polietileno tereftalato, em forma de rede protetora, com poros de $5 \mu \mathrm{m}$, que envolve o stent e que tem como finalidade prevenir a embolização de fragmentos da placa subjacente (Figura 1).

Descrição do stent SESAME ${ }^{\oplus}$ (Advanced Bio Prosthetic Surfaces Ltd., Texas, Estados Unidos)

Plataforma e polímero: Stent não-farmacológico, composto por plataforma de nitinol, compatível com o cateter $7 \mathrm{Fr}$, associada a sistema de inserção e bainha retrátil, que, mobilizados no momento do implante, promovem a liberação da prótese auto-expansível. A membrana microporosa para proteção contra eventos embólicos que reveste o stent é também de nitinol, com poros de $5 \mu \mathrm{m}$ (Figura 1).

\section{População avaliada}

Foram incluídos neste estudo 11 pacientes com lesões de novo em PS, tratados com stent MGuard ${ }^{\circledR}$ como parte do estudo INSPIRE, conduzido em nossa instituição entre novembro de 2007 e maio de 2008. Em resumo, o estudo INSPIRE é um estudo tipo firstin-human, não-randomizado, de braço único, com inclusão consecutiva de pacientes (inclusão em andamento) em uma única instituição (Instituto Dante Pazzanese de Cardiologia - São Paulo, SP). O estudo tem como objetivo investigar o desempenho e a eficácia do stent $M G$ uard ${ }^{\circledR}$ no tratamento percutâneo de lesões em PS ou em artérias coronárias com carga trombótica significativa (este último subgrupo foi excluído da presente análise). Como grupo controle, analisamos e comparamos uma população similar de 14 pacientes do registro SESAME, realizado em nossa instituição entre 2005 e 2006. O registro SESAME foi um estudo tipo first-in-human, multicêntrico, com inclusão consecutiva de pacientes portadores de lesões de novo $(\geq 50 \%)$ em PS nas diferentes apresentações clínicas, objetivando avaliar a eficácia e a segurança tardia do stent auto-expansível SESAME ${ }^{\circledR}$. Os pacientes incluídos nos dois estudos estão em adequação com a Declaração de Helsinki relacionada à investigação em humanos, e os protocolos foram aprovados pelo Comitê de Ética da instituição. Todos os pacientes incluídos assinaram termo de consentimento antes da inclusão no estudo. 


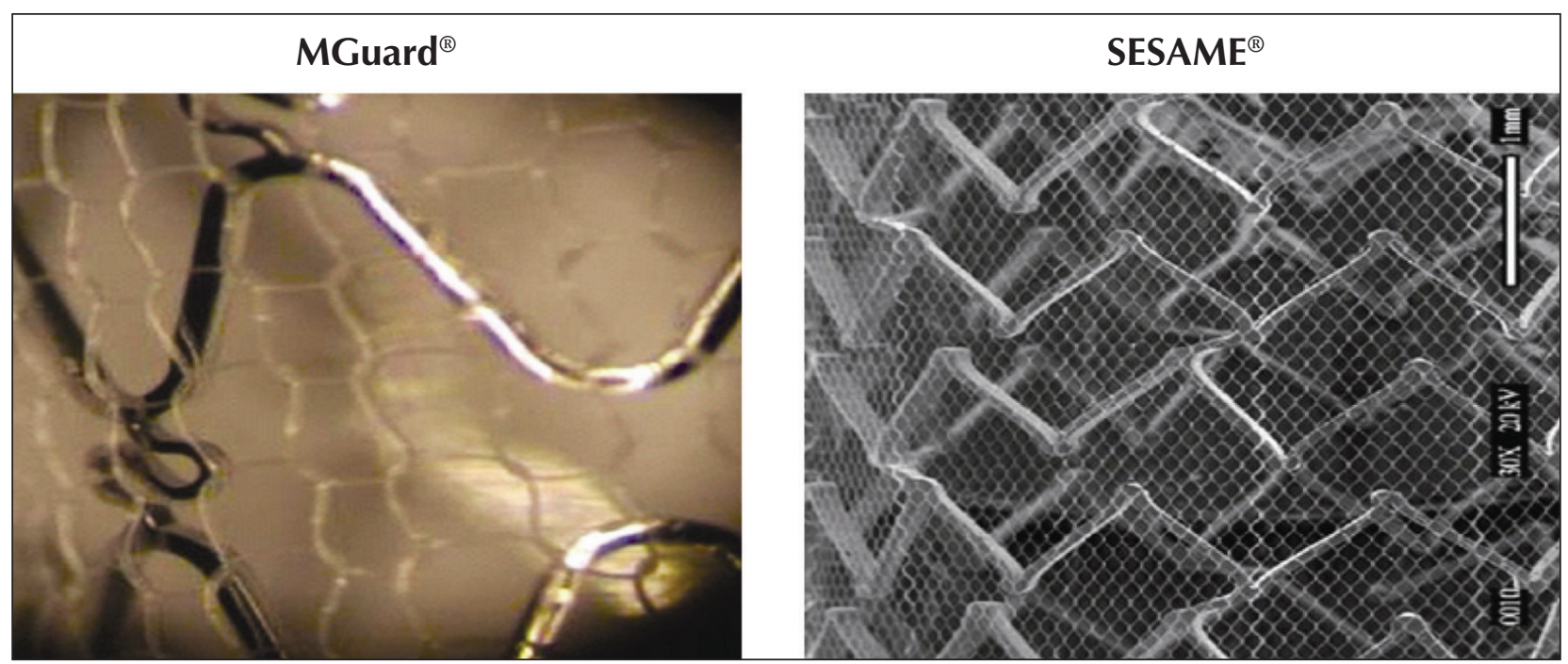

Figura 1 - À esquerda, stent MGuard ${ }^{\circledR}$ (InspireMD Ltd.,Tel Aviv, Israel); à direita, stent SESAME ${ }^{\circledR}$ (Advanced Bio Prosthetic Surfaces Ltd., Texas, Estados Unidos).

\section{Procedimento e protocolo medicamentoso adjunto}

As ICPs foram realizadas de acordo com as diretrizes atuais e a estratégia utilizada para abordar as lesões em PS foi determinada, principalmente, pela característica ímpar de os dispositivos MGuard ${ }^{\circledR} \mathrm{e}$ SESAME ${ }^{\circledR}$ não necessitarem o uso combinado de filtros de proteção distal e promoverem liberação balãoexpansível e auto-expansível, respectivamente. Os stents MGuard $^{\circledR}$ estavam disponíveis nos diâmetros de 2,5 mm a 4,0 mm e na extensão de $12 \mathrm{~mm}$ a $39 \mathrm{~mm}$, enquanto os stents SESAME ${ }^{\circledR}$ estavam disponíveis nos diâmetros de 3,0 mm a 5,0 mm e na extensão de $20 \mathrm{~mm}$. Ao operador coube decidir pela realização ou não de pré- e pós-dilatação, e pelo uso de inibidores de glicoproteína IIb/IIla intraprocedimento. O protocolo antitrombótico foi similar em ambos os grupos e consistia na administração de dois agentes antiplaquetários: aspirina (ácido acetilsalicílico - AAS) e um tienopiridínico. O pré-tratamento (AAS 200 mg e clopidogrel 300-600 mg) foi realizado 24 horas antes da intervenção. Após a intervenção, a terapia com AAS era mantida indefinidamente, na dose de $100 \mathrm{mg} /$ dia, e o clopidogrel, na dose de $75 \mathrm{mg}$ diários, era mantido por seis meses nos pacientes tratados com stent MGuard ${ }^{\circledR}$ e por três meses nos tratados com stent SESAME ${ }^{\circledR}$. Durante o procedimento, foi administrada heparina endovenosa (70-100 unidades/kg) para manter o tempo de coagulação ativado $>250$ segundos (ou > 200 segundos no caso de ser administrado inibidor da glicoproteína llb/ IIla). Eletrocardiograma (ECG) com 12 derivações foi obtido de rotina antes do procedimento, imediatamente após o procedimento e depois de 24 horas. Exames laboratoriais de rotina incluíram enzimas cardíacas (CPK e CkMB) pré-procedimento (< 24 horas), 18-24 horas pós-procedimento, e diariamente até a alta hospitalar em caso de alteração das mesmas.

\section{Análise por angiografia coronária quantitativa}

A angiografia coronária quantitativa (ACQ) do vasoalvo foi realizada pré- e pós-procedimento, após a administração intracoronária de nitrato (50-200 $\mu \mathrm{g})$, em pelo menos duas projeções ortogonais. As imagens foram armazenadas em formato digital e analisadas utilizando-se um sistema de detecção automática de bordas (QCA-CMS, Medis Medical Imaging System, Nuenen, Holanda), e a ponta do cateter-guia foi totalmente preenchida por contraste, para calibração do dispositivo.

A análise morfológica qualitativa da lesão foi feita de acordo com os critérios padrão de complexidade definidos pelo sistema de classificação do American College of Cardiology/American Heart Association ${ }^{4}$; a análise do enxerto venoso aortocoronário incluiu a avaliação da distribuição de placas ateroscleróticas e trombos, classificando as PS como "degeneradas", quando houvesse acometimento de pelo menos dois terços de sua extensão por material aterotrombótico, e "não-degeneradas", na ausência dessas características. TIMI Frame Count (TFC) ${ }^{5}$ foi realizado para mensurar o fluxo miocárdico pós-procedimento desses enxertos venosos aortocoronários.

A análise de ACQ foi realizada após o procedimento (off-line) por um investigador independente, e incluiu a avaliação dos seguintes parâmetros relacionados à lesão-alvo: 1) diâmetro de referência do vaso (DRV); 2) diâmetro luminal mínimo (DLM); 3) extensão da lesão; 4) diâmetro de estenose (DE); e 5) expansão do stent (ES), calculado como diâmetro do stent (DS)/DRV.

\section{Análise por ultra-som intracoronário}

As imagens do ultra-som intracoronário (USIC) pósprocedimento foram adquiridas utilizando-se transdutor 
de elemento único, rotacional, com freqüência de 40 $\mathrm{MHz}$, envolto em uma bainha com perfil 2,6 Fr, com recuo motorizado em sistema de tração automática à velocidade de $0,5 \mathrm{~mm} / \mathrm{s}$ e conectado a um scanner comercial (Galaxy 2, Boston Scientific Corp., Nattick, MA, Estados Unidos). As imagens foram digitalizadas para análise quantitativa off-line, de acordo com os critérios do American College of Cardiology Clinical Expert Consensus Document on IVUS6, por um programa de planimetria computadorizada comercialmente disponível (EchoPlaque 2, INDEC Systems Inc., Mountain View, CA, Estados Unidos). As áreas do vaso (AV) e do stent (AS) foram determinadas, a cada milímetro, por planimetria computadorizada, e utilizadas para obter a expansão do stent: $\mathrm{ES}=$ média $\mathrm{AS} /$ média $\mathrm{AV}$.

\section{Desfechos, definições e seguimento clínico}

O desfecho primário desta análise foi o sucesso angiográfico e o sucesso do procedimento. Secundariamente, comparou-se a taxa combinada dos eventos adversos combinados (ECAM) de óbito cardíaco, IM e revascularização da lesão-alvo (RLA) entre os dois grupos no período hospitalar e ao final de trinta dias de seguimento. O sucesso angiográfico foi definido como: fluxo final $\mathrm{TIMI}=3$, estenose residual $<20 \%$ pela ACQ, e ausência de complicações agudas como dissecção, perfuração e fenômenos de slow flow e no-reflow. O sucesso do procedimento foi definido pelo sucesso angiográfico na ausência de ECAM na fase hospitalar. Como regra, todos os óbitos foram considerados cardíacos, a não ser que uma causa não-cardíaca pudesse ser claramente estabelecida por estudo clínico ou patológico. O diagnóstico de IM foi baseado no aparecimento de nova ondaQ patológica em duas ou mais derivações contíguas no ECG e/ou elevação da CkMB > 3 vezes o limite superior da normalidade. Trombose de stent foi definida de acordo com as proposições do Academic Research Consortium ${ }^{7}$.

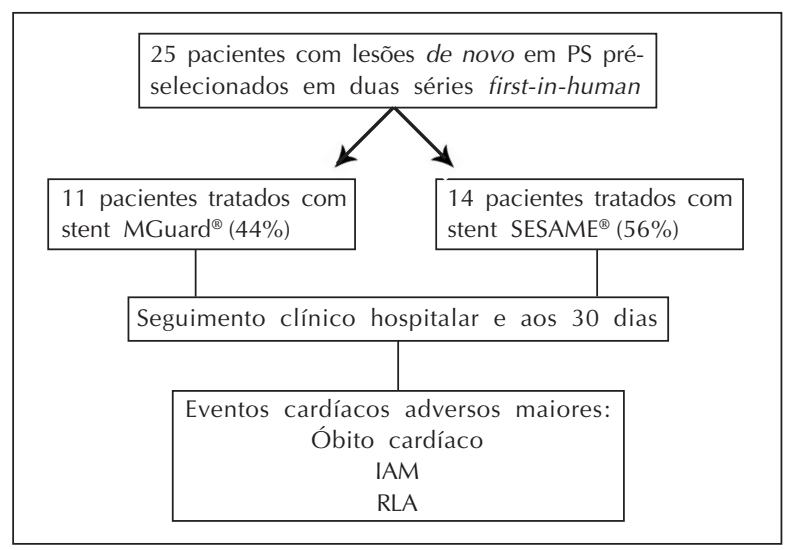

Figura 2 - Fluxograma de inclusão de pacientes.

IAM = infarto agudo do miocárdio; PS = ponte de safena; RLA = revascularização da lesão-alvo.
O seguimento clínico foi realizado trinta dias após o procedimento, e consistiu de visita médica agendada ou contato telefônico, realizado segundo protocolo pré-definido.

\section{Análise estatística}

As variáveis contínuas estão apresentadas como média \pm desvio padrão e foram comparadas pelo teste $t$ de Student não-pareado para amostras de distribuição normal. As variáveis categóricas estão reportadas como números absolutos e porcentagens (\%) e foram comparadas pelo teste do qui-quadrado de Pearson ou teste exato de Fisher, quando apropriado. Considerou-se estatisticamente significante $p<0,05$ (bicaudal). As análises foram realizadas com o auxílio do programa estatístico SPSS versão 16.0 (SPSS Inc., Chicago, IL, Estados Unidos).

\section{RESULTADOS}

\section{Características clínicas, angiográficas e ultra-sonográficas}

A Figura 2 apresenta o fluxograma de inclusão dos pacientes. Os pacientes dos dois grupos apresentavam perfis clínicos de complexidade superponíveis e idade dos enxertos venosos aortocoronários equivalente $\left(9,7 \pm 6,2\right.$ anos no grupo MGuard ${ }^{\circledR}$ vs. 10,1 $\pm 3,8$ anos no grupo SESAME $\left.{ }^{\circledR} ; \mathrm{p}=0,80\right)$, conforme demonstrado na Tabela 1. A média das idades foi de 69 anos, houve predomínio do sexo masculino em ambos os grupos, um quarto dos pacientes era portador de diabetes e quase a metade deles foi tratada na vigência de síndrome coronária aguda.

O enxerto venoso para a artéria descendente anterior foi o vaso-alvo mais freqüentemente tratado nos dois grupos $\left(45,5 \%\right.$ no grupo MGuard ${ }^{\circledR}$ vs. $42,8 \%$ no grupo SESAME ${ }^{\circledR} ; p=0,62$ ). Conforme a classificação morfológica adotada para avaliar o aspecto angiográfico das PS, a maioria apresentava enxertos classificados como degenerados (91\% no grupo MGuard ${ }^{\circledR}$ vs. $86 \%$ no grupo SESAME $\left.{ }^{\circledR} ; p=0,51\right)$.

A estratégia de ICP com implante de stent dedicado para abordar PS degeneradas foi similar em ambos os grupos, exceto pela maior necessidade de pós-dilatação do stent auto-expansível SESAME ${ }^{\circledR}(78,6 \%$ vs. 63,6\% no grupo MGuard $\left.^{\circledR} ; p=0,30\right)$. O grupo de pacientes tratados com stent MGuard ${ }^{\circledR}$ utilizou cerca de três vezes menos glicoproteína Ilb/IIla intraprocedimento que o grupo SESAME $^{\circledR}(9,0 \%$ vs. $21,4 \%$, respectivamente; $p=0,05)$. A mensuração do TFC evidenciou fluxo miocárdico preservado em ambos os grupos ao final do procedimento $(13,9 \pm 3,7$ vs. $18,7 \pm 8,5$, respectivamente; $p=$ $0,38)$. A Tabela 2 apresenta os dados angiográficos e do procedimento. O sucesso angiográfico pós-procedimento foi atingido em $100 \%$ dos casos nos dois grupos, porém com melhor acomodação e expansão final do stent observadas no grupo MGuard $^{\circledR}$, de acordo com análises de ACQ e USIC, demonstradas na Tabela 3. 
TABELA 1

Características clínicas basais

\begin{tabular}{|c|c|c|c|}
\hline Variável & MGuard $^{\oplus}(n=11)$ & $\operatorname{SESAME}^{\circledast}(n=14)$ & Valor de $p$ \\
\hline Média das idades, anos & $69,4 \pm 11,1$ & $68,9 \pm 9,1$ & 0,85 \\
\hline Sexo masculino, \% & $72,7(8)$ & $71,4(10)$ & 0,78 \\
\hline Diabetes melito, \% & $27,3(3)$ & $28,6(4)$ & 0,68 \\
\hline Hipertensão, \% & $100,0(11)$ & $100,0(14)$ & NA \\
\hline Dislipidemia, \% & $81,8(9)$ & $78,6(11)$ & 0,61 \\
\hline Tabagismo atual, \% & $27,3(3)$ & $28,6(4)$ & 0,68 \\
\hline História familiar de DAC, \% & $18,2(2)$ & $28,6(4)$ & 0,27 \\
\hline IAM prévio, \% & $63,6(7)$ & $64,3(9)$ & 0,82 \\
\hline Insuficiência renal*, \% & $18,2(2)$ & $21,4(3)$ & 0,58 \\
\hline Tempo de CRM, anos & $9,7 \pm 6,2$ & $10,1 \pm 3,8$ & 0,80 \\
\hline \multicolumn{4}{|l|}{ Apresentação clínica, \% } \\
\hline Angina estável & $45,5(5)$ & $42,8(6)$ & 0,60 \\
\hline Angina instável & $27,3(3)$ & $28,6(4)$ & 0,68 \\
\hline IAM & $18,2(2)$ & $14,3(2)$ & 0,45 \\
\hline Isquemia silenciosa & $9,0(1)$ & $14,3(2)$ & 0,40 \\
\hline
\end{tabular}

TABELA 2

Dados angiográficos e do procedimento

\begin{tabular}{|c|c|c|c|}
\hline Variável $(\mathrm{N}=25)$ & MGuard $^{\circledast}(n=11)$ & $\operatorname{SESAME}^{\circledast}(n=14)$ & Valor de $p$ \\
\hline \multicolumn{4}{|l|}{ Território miocárdico acometido } \\
\hline DA/Dg & $45,5 \%(5)$ & $42,8 \%(6)$ & 0,60 \\
\hline $\mathrm{Cx} / \mathrm{Mg}$ & $36,3 \%(4)$ & $35,7 \%(5)$ & 0,76 \\
\hline $\mathrm{CD}$ & $18,2 \%(2)$ & $21,4 \%(3)$ & 0,58 \\
\hline Pré-dilatação & $36,3 \%(4)$ & $35,7 \%(5)$ & 0,76 \\
\hline Pós-dilatação & $63,6 \%(7)$ & $78,6 \%(11)$ & 0,30 \\
\hline Pressão de liberação do stent, atm & $12,1 \pm 3,4$ & - & - \\
\hline Glicoproteína IIb/IIla & $9,0 \%(1)$ & $21,4 \%(3)$ & 0,05 \\
\hline Volume de contraste, ml & $85,2 \pm 12,2$ & $103,4 \pm 15,7$ & 0,34 \\
\hline TIMI Frame Count (TFC) & $13,9 \pm 3,7$ & $18,7 \pm 8,5$ & 0,38 \\
\hline Sucesso angiográfico & $100,0 \%(11)$ & $100,0 \%(14)$ & NA \\
\hline
\end{tabular}

\section{Desfechos intra-hospitalares e aos trinta dias}

Na fase hospitalar, observaram-se dois IM não-Q no grupo tratado com o stent SESAME ${ }^{\circledR}(14,3 \%$ vs. $0 \%$ no grupo MGuard $\left.^{\circledR} ; p=0,04\right)$. Após a alta hospitalar, ainda dentro do primeiro mês de evolução, um paciente do grupo SESAME ${ }^{\circledR}$ necessitou novo procedimento de RLA. Ao final de trinta dias, a taxa de ECAM foi, portanto, de $21,4 \%$ no grupo SESAME ${ }^{\circledR}$ e de $0 \%$ no grupo MGuard $^{\circledR}$ $(p=0,03)$. A Tabela 4 apresenta os resultados clínicos na fase hospitalar e aos trinta dias de seguimento.

\section{DISCUSSÃO}

Os bons resultados imediatos do presente estudo confirmam a exeqüibilidade dos stents dedicados ao tratamento de lesões ateroscleróticas em PS. Ainda, o emprego de stents balão-expansíveis $\left(\right.$ MGuard $\left.^{\circledR}\right)$ pare- 
TABELA 3

Angiografia coronária quantitativa e ultra-som intracoronário $(\mathrm{N}=25)$

\begin{tabular}{|c|c|c|c|c|c|c|}
\hline \multirow[b]{2}{*}{ Variável } & \multicolumn{3}{|c|}{ Pré-procedimento } & \multicolumn{3}{|c|}{ Pós-procedimento } \\
\hline & $\begin{array}{c}\text { MGuard }^{\circledR} \\
(n=11)\end{array}$ & $\begin{array}{c}\text { SESAME }^{\circledR} \\
(n=14)\end{array}$ & $\mathbf{p}$ & $\begin{array}{c}\text { MGuard }^{\circledR} \\
(n=11)\end{array}$ & $\begin{array}{c}\text { SESAME }^{\circledR} \\
(n=14)\end{array}$ & $\mathbf{p}$ \\
\hline Extensão da lesão, mm & $12,35 \pm 5,07$ & $13,28 \pm 4,85$ & 0,75 & - & - & - \\
\hline $\mathrm{DRV}, \mathrm{mm}$ & $3,02 \pm 0,58$ & $3,19 \pm 0,44$ & 0,68 & - & - & - \\
\hline $\mathrm{DLM}, \mathrm{mm}$ & $0,88 \pm 0,26$ & $0,90 \pm 0,18$ & $>0,99$ & $2,79 \pm 0,50$ & $2,55 \pm 0,44$ & 0,44 \\
\hline $\mathrm{DE}, \%$ & $71,48 \pm 8,09$ & $72,39 \pm 10,76$ & 0,80 & $8,95 \pm 3,77$ & $19,21 \pm 4,85$ & 0,15 \\
\hline $\mathrm{ES}, \mathrm{ACQ}$ & - & - & - & $0,87 \pm 0,22$ & $0,68 \pm 0,21$ & 0,08 \\
\hline ES, USIC & - & - & - & $0,51 \pm 0,13$ & $0,30 \pm 0,17$ & 0,06 \\
\hline
\end{tabular}

Valores são expressos como freqüência (\%) ou média \pm desvio padrão.

$\mathrm{ACQ}=$ angiografia coronária quantitativa; $\mathrm{DE}=$ diâmetro de estenose; DLM = diâmetro luminal mínimo; DRV = diâmetro de referência do vaso (PS); ES = expansão do stent; $\mathrm{N}=$ número de lesões; $\mathrm{n}$ = número de pacientes; USIC = ultra-som intracoronário.

TABELA 4

Resultados clínicos

\begin{tabular}{|c|c|c|c|c|c|c|}
\hline \multirow[b]{2}{*}{ Variável } & \multicolumn{3}{|c|}{ Resultados hospitalares } & \multicolumn{3}{|c|}{ Resultados em 30 dias } \\
\hline & $\begin{array}{c}\text { MGuard }^{\circledR} \\
(\mathrm{n}=11)\end{array}$ & $\begin{array}{c}\text { SESAME }^{\circledR} \\
(n=14)\end{array}$ & Valor de $p$ & $\begin{array}{c}\text { MGuard }^{\circledR} \\
(\mathrm{n}=11)\end{array}$ & $\begin{array}{c}\text { SESAME }^{\circledR} \\
(\mathrm{n}=14)\end{array}$ & Valor de $p$ \\
\hline ECAM & - & $14 \%(2)$ & 0,04 & - & $21 \%(3)$ & 0,03 \\
\hline Óbito cardíaco & - & - & NA & - & - & NA \\
\hline IM não-Q & - & $14 \%(2)$ & 0,04 & - & $14 \%(2)$ & 0,04 \\
\hline $\mathrm{IMQ}$ & - & - & NA & - & - & NA \\
\hline RLA & - & - & NA & - & $7 \%(1)$ & 0,09 \\
\hline
\end{tabular}

Valores são expressos como freqüência (\%) ou média \pm desvio padrão.

ECAM = eventos cardíacos adversos maiores; $I M=$ infarto do miocárdio; $n=$ número de pacientes; $N A=$ não se aplica; $R L A=$ revascularização da lesão-alvo.

ce ser mais efetivo, quando comparado ao de autoexpansíveis $\left(S E S A M E^{\circledR}\right)$, em relação à incidência de ECAM no primeiro mês de evolução.

As lesões ateroscleróticas em PS estão presentes na maioria dos enxertos com idade superior a cinco anos, sendo responsáveis por grande parte dos casos de IM nos pacientes revascularizados cirurgicamen$\mathrm{te}^{8,9}$. Nesse cenário, o tratamento percutâneo está associado a maior mortalidade aos trinta dias e seis meses, quando comparado ao tratamento de lesões coronárias em vasos nativos.

Nesse complexo subgrupo de pacientes e lesões, as taxas de IM periprocedimento chegam a $20 \%$ e são relacionados diretamente à embolização de material aterosclerótico para a microvasculatura distal, sendo este um preditor independente de pior prognóstico tardio, em particular de óbito cardíaco ${ }^{10,11}$.

Os elevados índices de insucesso associados ao procedimento percutâneo em PS têm motivado a bus- ca de soluções e dispositivos que diminuam e limitem a embolização distal de debris presentes nas lesões ateroscleróticas desses enxertos aortocoronários ${ }^{12,13}$. Tais lesões ateroscleróticas possuem características peculiares de maior risco e complexidade, em decorrência de placas mais friáveis, que pioram progressivamente com a idade da PS ${ }^{14,15}$.

Os dispositivos de proteção distal diminuíram efetivamente as taxas de mionecrose miocárdica durante o procedimento, como documentado no estudo SAFER ${ }^{16}$. Nesse estudo, a utilização de balão de proteção distal (Percusurge $^{\circledR}$ ) associado à aspiração do conteúdo de placa e trombo após a liberação do stent possibilitou redução relativa $(42 \%)$ de ECAM aos trinta dias.

Após o Percusurge ${ }^{\circledR}$, outros dispositivos de proteção distal e proximal foram testados em igual cenário, também se mostrando eficazes em reduzir microembolizações ${ }^{17,18}$ e eventos clínicos agudos e a longo prazo $^{19,20}$. Mais recentemente, uma metanálise publicada por Hillegass et al. ${ }^{21}$, envolvendo 3.430 pacientes com 
Mendes A, et al. Impacto dos Stents de Nova Geração no Tratamento de Enxertos Venosos Aortocoronários - Comparação Angiográfica e Ultra-sonográfica de Duas Séries First-in-Human: MGuard ${ }^{\circledR}$ vs. SESAME ${ }^{\circledR}$. Rev Bras Cardiol Invas. 2008;16(3):333-340.

lesões em PS tratados percutanemente, demonstrou que o uso de dispositivos de proteção distal nesse cenário foi protetor para mionecrose miocárdica.

Entretanto, nem todos os pacientes possuem características anatômicas compatíveis com o uso desses dispositivos. Em pesquisa realizada por Webb et al. ${ }^{22}$ incluindo 626 pacientes, 23\% não tinham condições anatômicas de utilizar quaisquer tipos de proteção (distal ou proximal), 39\% poderiam receber ambos os tipos, $18 \%$ poderiam receber somente proteção distal e $20 \%$, somente proteção proximal. Por outro lado, mesmo com seu uso, observou-se presença de embolização aterosclerótica distal clinicamente manifesta em $10 \%$ dos casos.

O desenvolvimento de stents revestidos com politetrafluoretileno (PTFE) poderia impedir a migração distal do conteúdo aterosclerótico, aumentando a segurança e a viabilidade do tratamento percutâneo nesse nicho de pacientes. No entanto, estudos randomizados prévios comparando stents revestidos com PTFE e stents convencionais (RECOVERS ${ }^{23}, \mathrm{STING}^{24}$, SYMBIOT III ${ }^{25}$ ) não conseguiram documentar a superioridade desses novos stents dedicados, tendo em vista os índices de ECAM, sobretudo IM e RLA, semelhantes entre os grupos durante seguimento hospitalar e tardio.

A presente análise traz a comparação de duas novas propostas de stents dedicados ao tratamento dos enxertos venosos aortocoronários. Enquanto o grupo $\mathrm{SESAME}^{\circledR}$ demonstrou taxas de IM não-Q periprocedimento mais elevadas durante seguimento intra-hospitalar, o grupo MGuard ${ }^{\circledR}$ cursou com a ausência de tais eventos aos trinta dias de seguimento. Essa diferença pode estar relacionada ao fato de o stent SESAME ${ }^{\circledR}$ auto-expansível ter maior perfil e necessitar mais freqüentemente procedimentos de pós-dilatação, aumentando as chances de deslocamento e embolização do material friável proveniente da placa aterotrombótica.

\section{Limitações do estudo}

A comparação entre dois novos dispositivos (MGuard $^{\circledR}$ e SESAME ${ }^{\circledR}$ ), realizada a partir da comparação de dois subgrupos pré-selecionados de séries firstin-human, submete esta análise às potenciais limitações de um estudo não-randomizado. A amostra limitada deste estudo impede conclusões definitivas a respeito do desempenho dos stents MGuard $^{\circledR}$ e SESAME $^{\circledR}$ em relação a desfechos clínicos relevantes. Um estudo multicêntrico, randomizado, controlado e com período maior de seguimento é necessário para a comparação definitiva entre esses dois stents de nova geração dedicados ao tratamento de PS degeneradas.

\section{CONCLUSÃO}

Nesta análise retrospectiva incluindo uma pequena amostra de pacientes pré-selecionados de duas séries first-in-human, o stent balão-expansível MGuard ${ }^{\circledR}$ foi superior ao stent auto-expansível SESAME ${ }^{\circledR}$ nos primeiros trinta dias de seguimento clínico.

\section{REFERÊNCIAS BIBLIOGRÁFICAS}

1. Jaegere PP, van Domburg RT, Feyter PJ, Ruygrok PN, van der Giessen WJ, Van den Brand MJ, et al. Long-term clinical outcome after stent implantation in saphenous vein grafts. J Am Coll Cardiol. 1996;28(1):89-96.

2. Bhargava B, Kornowski R, Mehran R, Kent KM, Hong MK, Lansky AJ, et al. Procedural results and intermediate clinical outcomes after multiple saphenous vein graft stenting. J Am Coll Cardiol. 2000;35(2):389-97.

3. Wong SC, Baim DS, Schatz RA, Teirstein PS, King SB $3^{\text {rd }}$, Curry RC Jr, et al. Immediate results and late outcomes after stent implantation in saphenous vein graft lesions: the multicenter U.S. Palmaz-Schatz stent experience. The Palmaz-Schatz Stent Study Group. J Am Coll Cardiol. 1995;26(3):704-12.

4. Stary HC, Chandler AB, Dinsmore RE, Fuster V, Glagov S, Insull $\mathrm{W}$ Jr. et al. A definition of advanced types of atherosclerotic lesions and a histological classification of atherosclerosis: a report from the Committee on Vascular Lesions of the Council on Arteriosclerosis, American Heart Association. Circulation. 1995;92(5):1355-74.

5. Gibson CM, Cannon CP, Daley WL, Dodge JT Jr, Alexander B Jr, Marble SJ, et al., for the TIMI 4 Study Group. TIMI frame count: a quantitative method of assessing coronary artery flow. Circulation. 1996;93(5):879-88.

6. Mintz GS, Nissen SE, Anderson WD, Bailey SR, Erbel R, Fitzgerald PJ, et al. American College of Cardiology Clinical Expert Consensus Document on Standards for Acquisition, Measurement and Reporting of Intravascular Ultrasound Studies (IVUS). A report of the American College of Cardiology Task Force on Clinical Expert Consensus Documents. J Am Coll Cardiol. 2001;37(5):1478-92.

7. Serruys PW, Daemen J. Are drug-eluting stents associated with a higher rate of late thrombosis than bare metal stents? Late stent thrombosis: a nuisance in both bare metal and drug-eluting stents. Circulation. 2007;115(11):1433-9.

8. Kugelmass AD, Sadanandan S, Cannon CP, Dibattiste PM, Robertson D, Braunwald E, for the TACTICS TIMI-18 Investigators. Early invasive strategy improves outcomes in acute coronary syndrome patients with prior CABG: Results from TACTICS-TIMI 18. Circulation. 2001;104(Suppl II):II-548.

9. Mathew V, Berger PB, Lennon RJ, Gersh BJ, Holmes DR Jr. Comparison of percutaneous interventions for unstable angina pectoris in patients with and without previous coronary artery bypass grafting. Am J Cardiol. 2000;86(9):931-7.

10. Kalon KL, Carrozza JP, Popma JJ. Creatine-kinase MB isoform (CK-MB) elevations following single vessel percutaneous revascularization of saphenous vein grafts. Circulation. 1998;98(Suppl I):I-353.

11. Hong MK, Mehran R, Dangas G, Mintz GS, Lansky AJ, Pichard $A D$, et al. Creatine-kinase-MB enzyme elevation following successful saphenous vein graft intervention is associated with late mortality. Circulation. 1999;100(24):2400-5.

12. Scheerder IK, Strauss BH, de Feyter PJ, Beatt KJ, Baur LH, Wijns W, et al. Stenting of venous bypass grafts: a new treatment modality of patients who are poor candidates for reintervention. Am Heart J. 1992;123(4 Pt 1):1046-54.

13. Strauss BH, Serruys PW, Bertrand ME, Puel J, Meier B, Goy $\mathrm{JJ}$, et al. Quantitative angiographic follow-up of the coronary Wallstent in native vessels and bypass grafts (European experience - March 1986 to March 1990). Am J Cardiol. 1992;69(5):475-81. 
14. Neitzel GF, Barboriak JJ, Pintar K, Qureshi I. Atherosclerosis in aortocoronary bypass grafts. Morphologic study and risk factor analysis 6 to 12 years after surgery. Arteriosclerosis. $1986 ; 6(6): 594-600$.

15. Walts AE, Fishbein MC, Sustaia H, Matloff JM. Ruptured atheromatous plaques in saphenous vein coronary artery bypass grafts: a mechanism of acute, thrombotic, late graft occlusion. Circulation. 1982;65(1):197-201.

16. Baim DS, Wahr D, George B, Leon MB, Greenberg J, Cutlip $D E$, et al. Saphenous vein graft Angioplasty Free of Emboli Randomized (SAFER) trial investigators. Randomized trial of a distal embolic protection device during percutaneous intervention of saphenous vein aorto-coronary bypass grafts. Circulation. 2002;105(11):1285-90

17. Stone GW, Rogers C, Hermiller J, Feldman R, Hall P, Haber $\mathrm{R}$, et al. Randomized comparison of the distal protection with a filter-based catheter and a balloon occlusion and aspiration system during percutaneous intervention of diseased saphenous vein aorto-coronary bypass grafts. Circulation. $2003 ; 108(5): 548-53$.

18. Baldus S, Köster R, Reimers J, Kähler J, Meinertz T, Hamm CW. Membrane-covered stents: a new treatment strategy for saphenous vein graft lesions. Catheter Cardiovasc Interv. $2001 ; 53(1): 1-4$.

19. Carrozza JP Jr, Mumma M, Breall JA, Fernandez A, Heyman E, Metzger C; PRIDE Study Investigators. Randomized evaluation of the TriActiv balloon-protection flush and extraction system for the treatment of saphenous vein graft disease. J Am Coll Cardiol. 2005;46(9):1677-83.
20. Rogers C. Proximal protection during saphenous vein graft intervention using the Proxis embolic protective system: a randomized, prospective, multicenter clinical trial (PROXIMAL). Presented at TCT 2005, Washington, D.C. 2004. J Am Coll Cardiol. 2006;44:1801.

21. Hillegass WB, Lyerly MJ, Patel NJ, Zoghbi GJ, Misra VK, Brott $\mathrm{BC}$. Frequency and predictors of post-procedural myonecrosis in saphenous vein graft intervention in the stent era: a metaregression. Circulation. 2006;114(Suppl II):II-733.

22. Webb JG, Carere RG, Virmani R, Baim D, Teirstein PS, Whitlow $\mathrm{P}$, et al. Retrieval and analysis of particulate debris after saphenous vein graft intervention. J Am Coll Cardiol. 1999;34(2):468-75.

23. Stankovic G, Colombo A, Presbitero $P$, van den Branden F, Inglese L, Cernigliaro C, et al. Randomized evaluation of polytetrafluoroethylene COVERed stent in Saphenous vein grafts. The Randomized Evaluation of polytetrafluoroethylene COVERed stent in Saphenous vein grafts (RECOVERS) Trial. Circulation. 2003;108(1):37-42.

24. Schächinger V, Hamm CW, Münzel T, Haude M, Baldus S, Grube $\mathrm{E}$, et al. A randomized trial of polytetrafluoroethylenemembrane-covered stents compared with conventional stents in aortocoronary saphenous vein grafts. J Am Coll Cardiol. $2003 ; 42(8): 1360-9$

25. Turco MA, Buchbinder M, Popma JJ, Weissman NJ, Mann T, Doucet S, et al. Pivotal, randomized U.S. study of the Symbiottrade mark covered stent system in patients with saphenous vein graft disease: eight-month angiographic and clinical results from the Symbiot III trial. Catheter Cardiovasc Interv. 2006;68(3):379-88. 